\title{
Multiple benign stromal cell tumours of the small bowel
}

\author{
J A Gall, R Chetty, A J Kemp, J C B Penfold
}

\begin{abstract}
A rare case of multiple small intestinal stromal cell tumours is described in a 79 year old woman who presented with melaena and anaemia. At surgery, about 40 lesions were noted on the serosal surface of the small intestine, the largest of these tumours being located in the midjejunum. This lesion showed central necrosis and haemorrhage with a sinus opening into the jejunal lumen. Histological examination of this jejunal tumour showed epithelioid and spindle shaped cells. A smaller biopsied tumour was a pure spindle cell lesion. Both lesions fitted the criteria for benign stromal cell tumours. Although skenoid fibres were identified, the immunophenotype was characteristically heterogenous. Multiple benign small intestinal tumours can raise the spectre of metastases when seen at surgery, although their presence in this case does not seem to indicate this.
\end{abstract}

(F Clin Pathol 1993;46:869-871)

Stromal cell tumours of the small bowel are rare and the presence of several of these tumours in the one patient is exceedingly rare. Although a review of the published cases indicates four reports of multiple tumours, ${ }^{1-4}$ very few clinical and pathological details were given. The tumours listed in these reports included leiomyomas, leiomyosarcomas, myomas, fibromas and neurogenic tumours. More recently, these and other spindle cell tumours of the gastrointestinal tract have been collectively referred to as stromal cell tumours due to their uncertain histogenesis and heterogenous immunophenotype. Criteria have been developed to assist in the differentiation between benign and malignant lesions based on their size, the degree of mitotic activity, the presence of necrosis, the cellularity and cellular atypia. Their clinical behaviour, however, remains unpredictable from the histological appearance.

The occurrence of multiple, benign-looking stromal cell tumours in the one patient raises the question of malignancy, particularly in the light of the unpredictable nature of these tumours.

Case report

A 79 year old woman with a history of having had a Billroth I gastrectomy presented after three episodes of melaena and associated dizziness. She did not have either postmenopausal bleeding or clinical features of von Recklinghausen's disease and associated conditions. There was no family history of neurofibromatosis. On examination, she was anaemic but vital signs were stable. Gynaecological examination was normal. Investigations showed that her haemoglobin concentration was initially $108 \mathrm{~g} / \mathrm{l}$ but this subsequently dropped to $90 \mathrm{~g} / \mathrm{l}$. Clotting time was normal. Endoscopy yielded normal results and a barium enema showed scattered diverticular disease, mainly in the sigmoid colon. Barium meal showed a small $5 \mathrm{~mm}$ persistent pool of barium in the distal small bowel overlying the left iliac wing. This was associated with an eccentric intramural mass causing deformity of the bowel lumen, the appearances of which were highly suggestive of an ulcerated leiomyoma. The patient was taken to theatre where a mass lesion was noted on the anterior mesenteric border of the mid-jejunum. About 40 smaller additional nodules (ranging from $5-40 \mathrm{~mm}$ in size) were also noted on the serosal mucosa of the small bowel. There was no evidence of parietal peritoneal seeding. A segmental resection of the mid-jejunum was performed and one further serosal nodule was biopsied. The impression was of multiple serosal metastases. The patient made a satisfactory recovery and was then discharged. Follow up after six months showed that she was well, with no evidence of recurrence or metastases.

\section{Pathological findings}

Macroscopically, the resection specimen comprised a $45 \mathrm{~mm}$ segment of jejunum with a $40 \times 30 \times 25 \mathrm{~mm}$ cream-coloured mass attached to the serosal surface. The mass contained fibrillary material and central haemorrhage. A sinus extended from the tumour into the jejunal lumen. The second biopsy specimen consisted of a firm, whitish nodule covered by a capsule and measured $13 \times 10 \times 9 \mathrm{~mm}$.

Histologically, the jejunal lesion comprised a well demarcated mass arising in the muscularis propria with a wide rim of epithelioid and spindle shaped cells arranged in fascicles (fig 1). Both types of cells contained pleomorphic, vacuolated nuclei with occasional nucleoli. There was a variable degree of cytoplasmic vacuolation. Mitoses were not identified. Scattered among the cells were eosinophilic globules resembling skenoid fibres. Chronic inflammatory cells, consisting 
predominantly of lymphocytes, were scattered throughout the lesion. The central and predominant portion of the mass was necrotic and haemorrhagic and had been converted into an organised haematoma. A sinus opening into the jejunal lumen was identified; this was lined by granulation tissue (fig 1). The overlying mucosa was not eroded or ulcerated. The smaller specimen consisted of uniform, spindle shaped cells arranged in fascicles without areas of degeneration. Skenoid fibres were present and the lesion lay partly within the muscularis propria. Neuritic processes and axons were not identified within the lesions using Bodian's stain.

Immunohistochemically, some but not all of the tumour cells in both specimens stained with vimentin, neurone specific enolase, S100 and smooth muscle actin. The cells did not stain with either neurofilament protein (68 kilodalton component) or desmin. The fibres of the muscularis propria (which served as an in-built control) stained strongly for smooth muscle actin and desmin. A few cells in the jejunal lesion stained positively with proliferating cell nuclear antigen (PCNA)

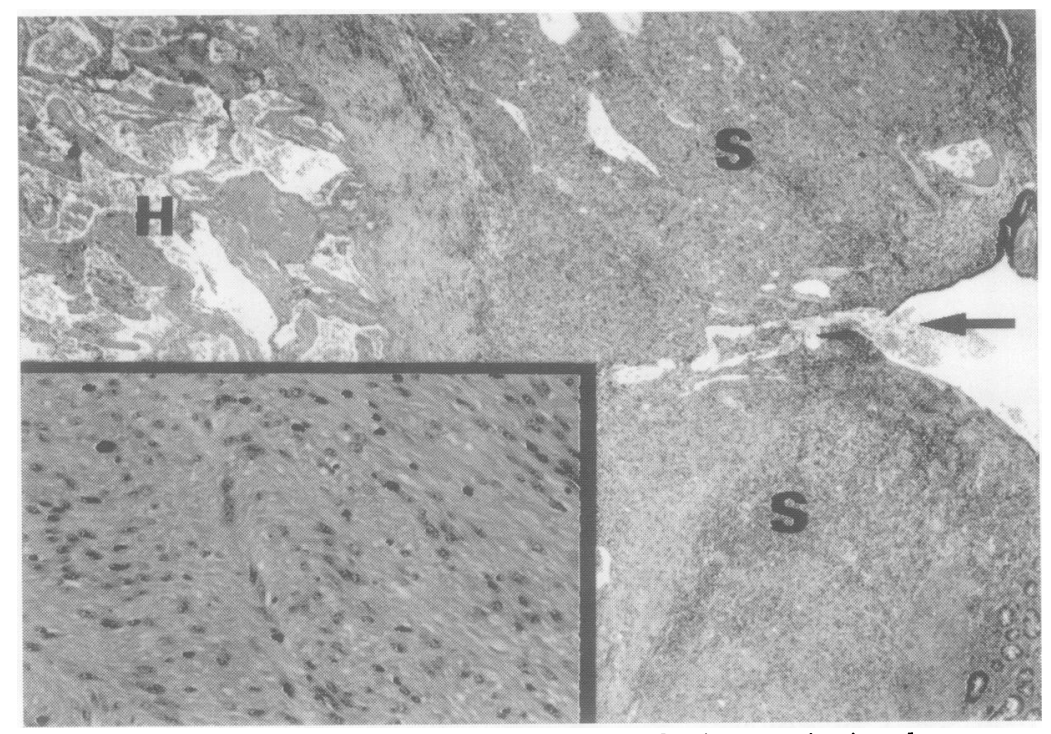

Figure 1 The jejunal stromal cell tumour $(S)$ showing the sinus opening into the gut lumen (arrow) and the central organised haematoma $(H)$. Inset: An area of the tumour showing spindle-shaped cells arranged in fascicles (haematoxylin and eosin).

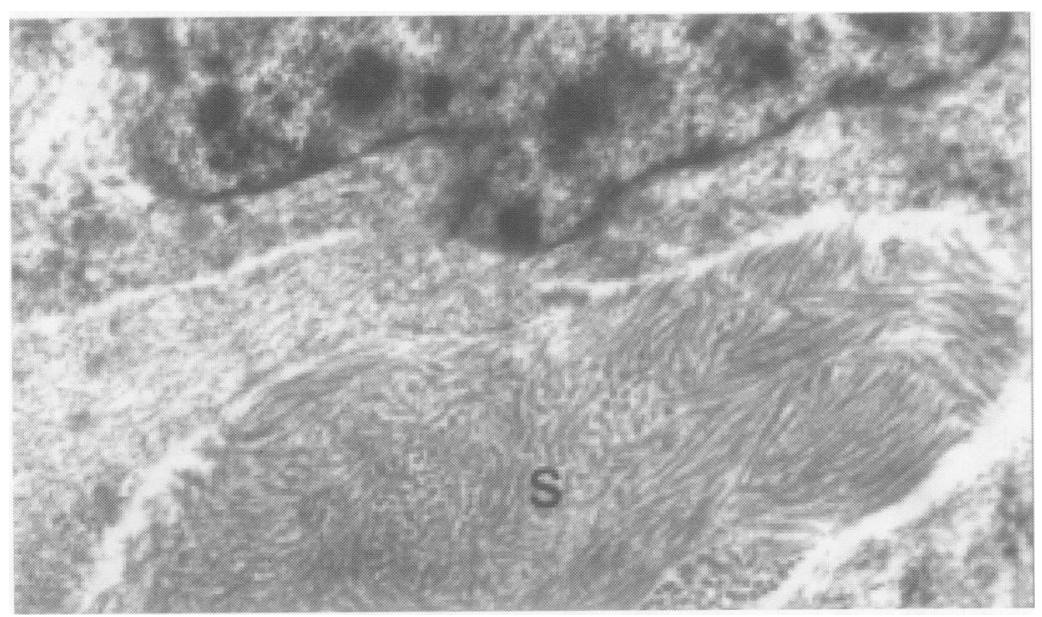

Figure 2 Electron micrograph showing the nucleus of a tumour cell and the interposed intercellular skenoid fibres $(S)$. using the monoclonal antibody PC10. A PC10 index, as described by $\mathrm{Yu}$ et $a l,{ }^{5}$ was estimated to be $0.003 \%$. The smaller specimen was unreactive for PC10 (PC10 index $0 \%$ ).

Ultrastructurally, the tumour cells were elongated with an ovoid nucleus, small nucleolus, and a few cytoplasmic organelles. Focally, between the cells, were skenoid fibres, often arranged in a nodular pattern (fig 2).

\section{Discussion}

Spindle cell tumours of the gastrointestinal tract have recently been collectively grouped as stromal cell tumours due to their uncertain histogenesis and heterogenous immunophenotype. Although criteria have been developed to assist in the differentiation between benign and malignant tumours, those that are not obviously malignant and have metastasised at the time of surgery are problematical for the pathologist. Increased mitotic activity, combined with the predominant cell type (spindle or epithelioid), generally seem to be the most reliable histological indicators. ${ }^{56}$ Other variables, however, have been reported as being useful and these have included tumour size, cellularity, degree of atypia, cellular and nuclear pleomorphism and the presence of necrosis. ${ }^{78}$ These criteria are not specific and what may be classified by one author as malignant is classified by another as benign or borderline. Very recently, DNA aneuploidy, ${ }^{6}$ PCNA immunostaining and nucleolar organiser region staining $^{5}$ have also been shown to correlate well with the histological grade.

The presence of multiple lesions raises the possibility of metastases. Using the criteria of cellularity, predominant cell type, and mitotic activity, 578 the two sampled lesions were benign. The size of the jejunal lesion, however, raises the possibility of a sarcomatous lesion under some criteria. ${ }^{78}$ The histological appearance and PC10 index for this lesion was, however, in the range for benign tumours. ${ }^{5}$ In view of the presence of multiple lesions and the fact that only two were sampled, it is difficult to exclude the possibility that one of the unsampled lesions is malignant. Against this, however, is the clinical picture of a disease free follow up period of six months, and the histological blandness of the two sampled lesions.

In keeping with previous reports, these tumours displayed a heterogenous immunophenotype. At both the light and electron microscopic levels, both lesions contained the recently described skenoid fibres, a newly recognised neurogenic spindle cell tumour marker. ${ }^{9}$ Skenoid fibres have been found in stromal tumours arising from the small intestine but not from other parts of the gastrointestinal tract and this has led to the hypothesis that small intestinal stromal tumours may be a unique group of tumours derived from neurogenic tissue. ${ }^{9}$ Neurofibromas also contain skenoid fibres, ${ }^{9}$ but the 
histological appearance and absence of axons in the current tumour, together with an absence of the clinical features of von Recklinghausen's disease, precludes a diagnosis of neurofibromatosis. These lesions could also represent a rare tumour analogous to leiomyomatosis. Such a case of leiomyomatosis involving the small intestine and colon has been reported, ${ }^{10}$ but in contrast to the current case, the tumours were diffuse intramural masses of smooth muscle cells which originated in the muscularis propria and muscularis mucosae and were unreactive immunohistochemically for vimentin, actin, and S-100.

The pathogenesis of multiple tumours is a matter of conjecture and most likely due to a field effect, with different tumours growing at different rates accounting for the disparity in size. The hormonal influence in the aetiology of multiple uterine leiomyomas is well known but does not seem to have a similar role here. Furthermore, the histogenesis of the intestinal lesions may be very different.

Although there have been previous reports $^{1-4}$ of patients with multiple stromal cell tumours in the small bowel, the details in these reports are very limited. Additionally, the classification of these tumours and the criteria for the differentiation between benign and malignant lesions have changed since these reports. Starr and Dockerty ${ }^{4}$ cited four cases of multiple tumours, two labelled as leiomyomas ( 0 mitoses/10 high power fields) and two as leiomyosarcomas (1 mitosis/10 high power fields). Under the current criteria for the differentiation between benign and malignant lesions, the two labelled as leiomyosarcomas would now probably be classified as benign. River et al listed seven cases. $^{3}$ Three of these were myomas and involved the entire small intestine. Two cases were fibromas, one of which involved the entire small intestine and the other showing 15 to 20 lesions within the jejunum and ileum. The final two cases were classified as neurogenic tumours involving the entire small intestine. The more recent reports presented individual cases in slightly greater detail. ${ }^{12}$ Han and Aldrete described the case of a 54 year old man who presented with a gastrointestinal bleed and was found to have multiple pedunculated or sessile leiomyomas with cystic degeneration, haemorrhage, and infarction. ${ }^{2}$ Most of the tumours measured $2-3 \mathrm{~cm}$ in maximum dimension with the largest measuring $8 \mathrm{~cm}$. Allen described a 60 year old man who presented with melaena and anaemia and was found to have two intraluminal leiomyomas in the jejunum, one measuring $6 \times 8 \mathrm{~cm}$ and the other $2.5 \times 2.0 \mathrm{~cm} .{ }^{1}$ Depending on the histological picture, the $8 \mathrm{~cm}$ lesion could, under some classifications, fall into the malignant category.

In conclusion, the real contention in stromal tumours is the distinction between benign and malignant. Histogenesis is a secondary factor that probably does not influence the behaviour. Clinicopathological correlation is required; no one histological variable can be reliably used, and histogenesis is inconclusive using immunohistochemical staining. The role of skenoid fibres in determining histogenesis points towards a neurogenic origin. ${ }^{9}$ This paper highlights the occurrence of multiple benign small intestinal tumours (most of which are asymptomatic), and which, at operation, can be worrying to the surgeon by raising the possibility of metastases.

1 Allen FA. Leiomyomata of gastrointestinal tract. $\mathcal{F}$ Kans Med Soc 1971;72:453-7.

2 Han SY, Aldrete JS. The radiology corner. Angiographic diagnosis of leiomyomas of the small intestine. $A m \mathcal{F}$ Gastroenterol 1977;68:91-4.

3 River L, Silverstein J, Tope JW. Benign neoplasms of the small intestine. International Abstracts of Surgery 1956; 102:1-38.

4 Starr GF, Dockerty MB. Leiomyomas and leiomyosarcomas of the small intestine. Cancer 1955;8:101-11.

5 Yu CCW, Fletcher CDM, Newman PL, Goodlad JR, Burton JC, Levison DA. A comparison of proliferating cell nuclear antigen (PCNA) immunostaining, nuclear organiser region (AgNOR) staining, and histological grading in gastrointestinal tumours. F Pathol 1992; 166:147-52.

6 Cooper PN, Quirke P, Hardy GJ, Dixon MF. A flow cytometric, clinical, and histological study of stromal neoplasms of the gastrointestinal tract. Am $\mathcal{f}$ Surg Pathol 1992;16:163-70.

7 Akwari OE, Dozois RR, Weiland LH, Beahrs OH. Leiomyosarcoma of the small and large bowel. Cancer 1978;42:1375-84.

8 Evans HL. Smooth muscle tumours of the gastrointestinal tract. Cancer 1985;56:2242-50.

$9 \mathrm{Min} \mathrm{K-W.} \mathrm{Small} \mathrm{intestinal} \mathrm{stromal} \mathrm{tumors} \mathrm{with} \mathrm{skenoid}$ fibres. Clinicopathological, immunohistochemical, and ultrastructural investigations. Am f Surg Pathol 1992; 16:145-55.

10 Vallaeys JH, Cuvelier CA, Bekaert L, Roels H. Combined leiomyomatosis of the small intestine and colon. Arch Pathol Lab Med 1992;116:281-3. 